Thresholds and $Q$ Values of

Nuclear Reactions Induced by

Neutrons, Protons, Deuterons,

Tritons, ${ }^{3} \mathrm{He}$ Ions, Alpha

Particles, and Photons

\author{
R. J. Howerton
}

March 25, 1981 


\title{
Thresholds and $\mathbf{Q}$ Values of Nuclear Reactions Induced by Neutrons, Protons, Deuterons, Tritons, ${ }^{3} \mathrm{He}$ Ions, Alpha Particles, and Photons
}

\author{
R. J. Howerton
}

March 25, 1981

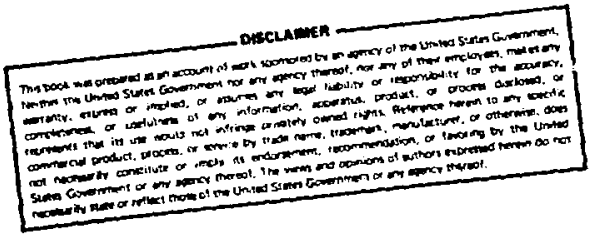




\section{FOREWORD}

The UCRL-50400 series, An Integrated System for Production of Neurror.rs and Photonics Calculational Constants, describes an integrated. computer-oriented system for the production and application of neutronics and photonics calculational constants.

The system supplies reliable, up-tp-date data, selects specific types of data on request, provides output in a variety of forms (ultimately in the form of input 10 other computer codes), and functions rapidly and cificiently.

The UCRL-50400 series comprises the following volumes:

- Vol. 1, Patt A, Rev. 3, ECSIL: a System for Storage, Resrieval, and Display of Experimental Neusron Data, September 1976.

- Vol. 1. Part B. Program ECSX4 (Version 78-1): Conversion of Experimentally Measured CrassSection Data from the Four-Center-Exchange $(X$-4) Format to the Livermore ESCIL Format, December 1978.

1976.

- Vol. 2. Rev. 2, A Bibliography of the Experimental Data of Neutron-Induced Interactions. July

- Vol. 3, Rev. 2, An Index of the Experimer:Ial Dara of Neutron-Induced Interactions, July 1976.

- Vol. 4, Evaluated Nucjear Cross-Section Library, April 1971.

- Vol. 5, Part A, Rev. J, CLYDE: A Code for the Production of Calculational Constants from Nuclear Data, September 1975.

- Vol. 5, Part B, Rev. 1, Relasivistic Transformations between Center-of-Mass and Laboratory Systems for Two-Bady Nuclear Reactions, April 1978.

December 1978.

- Vol. 6. Rev. 2, Tables and Graphs of Photon-Interaction Cross Sections from 1 keV to $100 \mathrm{MeV}$,

- Vol. 7. Part A. Rev. 1, Major Neutron-Induced Interactions $(Z<55)$ : Graphical, Experimental Data, July 1976.

- Vol. 7, Part B, Rev. 1, Major Neutron-Induced Interactions $(Z>55)$ : Graphical, Experimental Data, July : 976 .

- Vol. 6, Part A, Rev, 1, Supplemental Neutron-Induced Interactions $(Z<35)$ : Graphical, Experimental Data, July 1976.

- Vol. 8, Part B, Rev. I, Supplememtal Neutron-Induced Imeractions $(Z>35)$ : Graphical, Experimental Data, July 1976.

- Vol. 9, Thresholds of Nuclear Reactions Induced by Neutrons, Photons, Dew.erons. Tritons, and Alpha Particles, September 1970.

- Vol. 10, Rev. 1, Tabulated Experimental Data for Neutron-Induced Interactions, July 1976.

- Vol. 11, Experimental Data. Indexes, and Techniques of Obtaining a Selected Set of Neutron Resonance Parameters, May 1972.

- Vol. 12, An Allas of Resolved Neutron Kesonance Parameters, July 1972.

- Vol. 13, An Allas of Unresolved Neuiron Resonance Parameters, September 1972.

- Vol. 14, TARTNP: A Coupled Neutron-Photon Monte Carlo Trarsport Code, February 1976.

- Vol, 15, Part A, The LLL Evaluated-Nuclear Data Library (ENDL): Evaluation Techniques, Reaction Index, and Descriptions of Individual Evaluations, September 1975.

- Vol. 15, Part B, Rev. 1, The LLL Evaluated-Nuclear-Data Library (ENDL): Graphs of Cross Sections from the Library, October 1978.

- Vol. 15, Part C. The LLL Evaluated-Nuclear-Data Library (ENDL): Transtation of ENDL Neutron-Induced Interaction Data into the ENDF/B Fomat. April 1976.

- Vol. 15, Part D, Rev. 1. The LLL Evaluated-Nuclear-Data Libiary (ENDL): Descripticns of Individual Evaluations for $Z=0.98$, May 1978.

1979.

- Vol. 15, Part E, Data Testing Results for the LLL Nuclear Data Library (ENDL-78), August

- Vol. 15, Part F, Experimental and Evaluated Elastic Nuclear Plus Interference Cross Sections for Light Charged Partisles, July 1980. 
- Vol. 16, Rev. 2, Tabular ard Graphical Presentation of 175 Neutron-Group Constants Derived from the LLL Evaluated-Nuclear-Daja Librany (ENDL), October 1978.

- Vol. 17, Part A, Rev. 2, Proznam LINEAR (Version 79-1): Linearize Data in the Evaluated Nuclear Dafa File/Version $B$ (ENDF/D) Format, October 1979.

- Vol. 17, Part B, Rev. ż, Prozram SIGMAI (Version 79.1): Doppler Broaden Ewalwated Cross Secrions in the Evaluared Nuclear Data File/Version B (ENDF/D) Format, October 1979.

- Vol. 17, Part C, Program RECENT: Reconstruction of Energy-Dependent Cross Sections in Resonance Parameters in the ENDF/B Fommat, October 1979.

- Vol. 17, Part D, Program GROUPIE: Calculation of Self-Shielded Cross Sections and Multiband Parameters from Evaluated Dala in the ENDF/B Format, 1980.

- Vol. 17, Part E, Program EVALPLOT: Plot Data in the Evaluated-Nuclear-Data FilefVersion $E$ (ENDF/B) Format, February 1979. January 1979.

- Vol. 17, Part F, DOWNER (Version 79-1): Group Collapse Cross Section and Transfer Matrices,

- Vol. 18, ACTL: Evaluated Neutron Activation Cross-Secrion Library, October 1978.

1977.

- Vol. 19, Neutron-Induced Angular and Energy Distributions: Graphical Experimertal Data, April

- Vol. 20, Bonderenko Self-Shielded Cross Sections and Multiband Parameters Derived from the LLL Evaluated-Nuclear-Data Library (ENDL), July 1978.

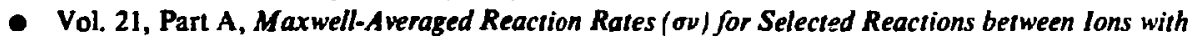
Atomic Mass < 11, February 1979.

- Vol. 21, Part C, Program SIGMAL (Versian 79-1): Doppler-Broaden Evaluated Cross Sections in the Livermore-Evaluated Nuclear Data Library (ENDL) Format, March 1979.

- Vol. 22, GAMIDENT: A Program to Aid in the Identification of Unknown Materials by GammaRay Spectroscopy, January 1980.

- Vol. 23, ENSL and CDRL: Evaluated Nuclear Structure Libraries, February 1981.

- Vol. 24, Thresholds and $Q$ Values of Nuclear Reactions Induced by Neutrons. Protons, Deuterons.

Tritons. ${ }^{3}$ He Ions, Alpha Particles, and Photons, Marsh 1981. 
CONTENTS

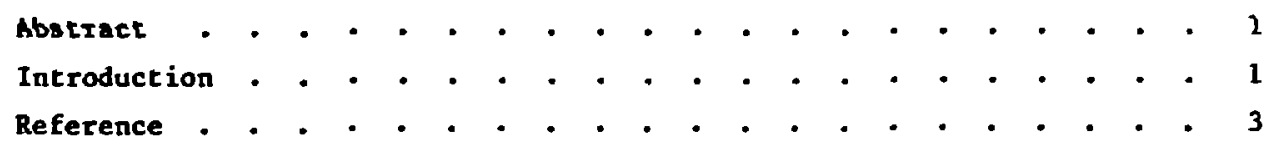




\section{THRESHOLDS AND $Q$ VALUES OF MUCLEAR REACTIOHS INDUCED BY NEUTRONS, PROTONE, DEUTERONS, TRITONS, ${ }^{3}$ He IONS, ALPHA PARTICLES, AND PHOTONS}

\section{ABSTRACT}

I have used the 1977 Wapstra and Bos nuclear mass data tables to derive tables for thresholds and $Q$ values of nuclear reactions induced by neutrons, protons, deuterons, tritons, ${ }^{3}$ He ions, alpha particles, and photons.

\section{INTRODUCTION}

I have used the 2977 Wapstra and Bos nuclear mass data tables ${ }^{1}$ to derive tables for thresholds and $Q$ values of nuclear reactions induced by neutrons, protons, deuterons, tritons, 3 He ions, alpha particles, and photons. These tables are displayed on the microfiche included with this report.

The threshold of a reaction is defined as the minimum kinetic energy of an impinging particle that makes the reaction energetically possible. For reactions induced by charged incident particles, the real threshold, i.e., the energy at which the reaction has a nonzero cross section, will be at a value somewhat greater than the mass-difference threshold because of coulomb effects-especially if the coulomb barrier is the same or greater than the mass-difference threshold. It is not possible to give a precise value to the real threshold because of tunneling effects. Consequently, the values presented in the tables for thresholds are derived from mass differences only.

The tables for thresholds have three different types of entries: positive entries, negative entries, and dashes. A positive entry is the mass-difference threshold; a negative entry, the negative of the positive 9 value associated with an exoergic reaction, indicates an exoergic reaction with a zero threshold. A dashed entry indicates that the mass of the residual nuelei is not known or, in the case of very light nuclei, that the reaction is not possitle. For example, a neutron impinging on a deuteron cannot initiate a reaction in which an alpha particle is eaitted. 
The 9 values presented here are defined as the sum of the masses of all particles in the exit channel subtracted from the sum of the masses of all particles in the entrance channel. This difference is multiplied by $931.502 \mathrm{MeV} / \mathrm{am}$ to convert to megaelectron volts.

To calculate the thresholds, I used the following assumption: if the mas of the impinging particle is $m$, then the threshold for reactions having negative $Q$ values is $-[(A+m) / A] Q$, where $A$ is the mass of the target. 


\section{REFERENCE}

1. A. H. Hapstra and K. Bos, "The 1977 Atomic Mass Evaluation in Four Parts," At. Data Hucl. Data Tables 19, 177-214 (1977). 